Document downloaded from:

http://hdl.handle.net/10251/43259

This paper must be cited as:

Garcia March, MA.; Gimenez Palomares, F.; Villatoro, FR.; Pérez Quiles, MJ.; Fernández De Córdoba Castellá, PJ.; Fernández De Córdoba (2011). Unisolvency for Multivariate Polynomial Interpolation in Coatmèlec Configurations of Nodes. Applied Mathematics and Computation. 217(18):7427-7431. doi:10.1016/j.amc.2011.02.034.

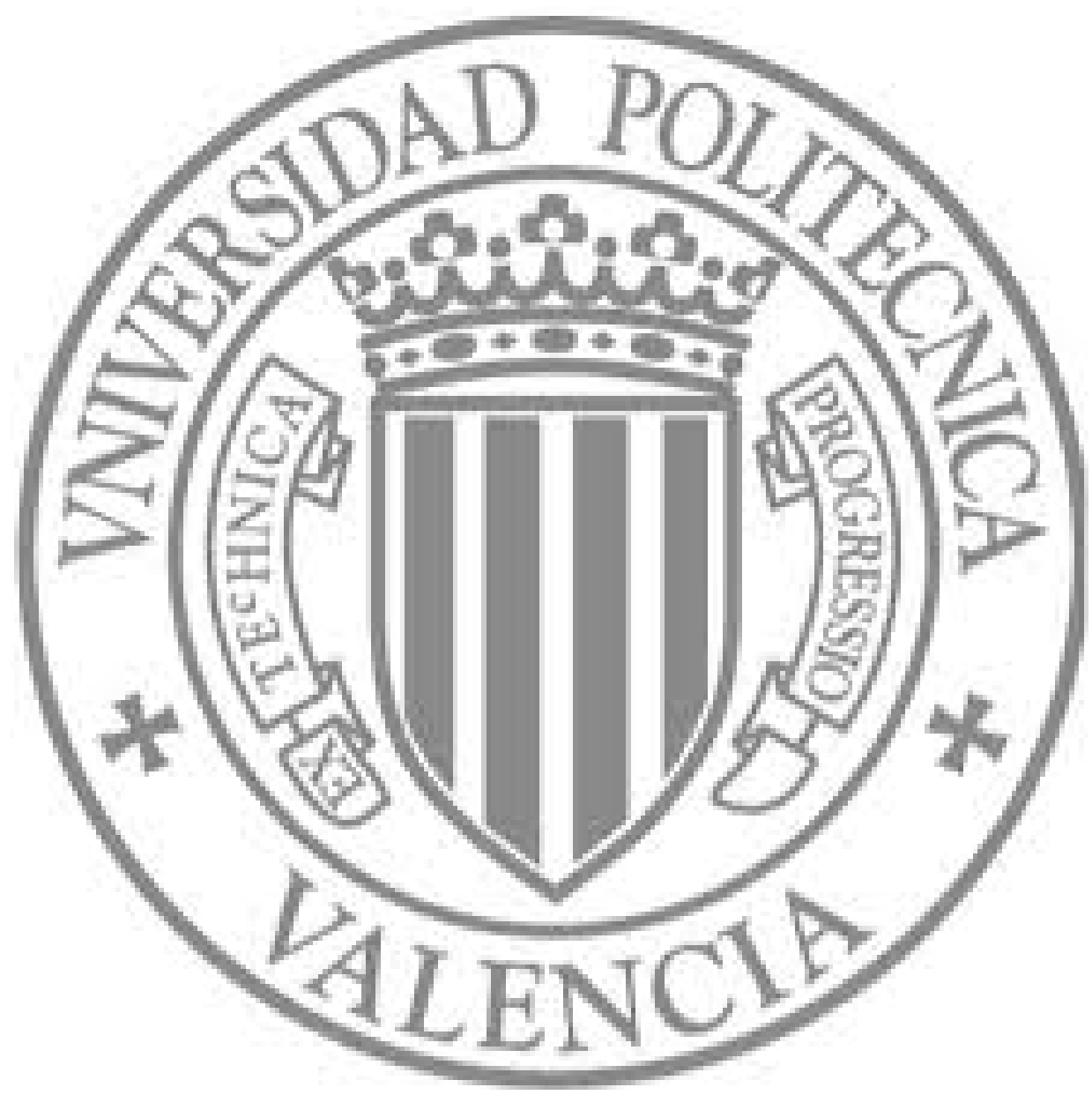

The final publication is available at

http://dx.doi.org/10.1016/j.amc.2011.02.034

Copyright Elsevier 


\title{
Unisolvency for Multivariate Polynomial Interpolation in Coatmèlec Configurations of Nodes
}

\author{
Miguel-Ángel García-March a , Fernando Giménez ${ }^{\text {b }}$, \\ Francisco R. Villatoro ${ }^{c}$, Jezabel Pérez ${ }^{\text {b,* }}$, and \\ Pedro Fernández de Córdoba ${ }^{b}$ \\ a Department of Physics, Colorado School of Mines, Golden, Colorado (USA) \\ ${ }^{\mathrm{b}}$ Instituto Universitario de Matemática Pura y Aplicada - IUMPA. Universidad \\ Politécnica de Valencia. Valencia (SPAIN) \\ ${ }^{\mathrm{c}}$ Departamento de Lenguajes y Ciencias de la Computación \\ E.T.S.I. Industriales, Universidad de Málaga \\ Málaga (SPAIN)
}

\begin{abstract}
A new and straightforward proof of the unisolvability of the problem of multivariate polynomial interpolation based on Coatmèlec configurations of nodes, a class of properly posed set of nodes defined by hyperplanes, is presented. The proof generalizes a previous one for the bivariate case and is based on a recursive reduction of the problem to simpler ones following the so-called Radon-Bézout process.
\end{abstract}

Key words: multivariate interpolation, properly posed set of nodes, geometric characterization, Coatmèlec lattices

\section{Introduction}

The problem of polynomial interpolation of one-dimensional data has a widely known solution. However, despite its apparent simplicity, multivariate polynomial interpolation remains a topic of current research [1-3]. The existence and uniqueness of the interpolation polynomial strongly depends on the geometrical distribution of the interpolation points. The distribution of points

\footnotetext{
* Corresponding author. Tel: (+34) 963-877-007 (Ext. 76648)

Email address: jperezq@mat.upv.es (Jezabel Pérez).
} 
for which the interpolation problem is unisolvable is referred to as properly posed set of nodes (PPSN).

The mathematical characterization of the most general PPSN is not currently known. The configurations of nodes based on algebraic varieties, such as those of Bos [4] and Liang et al. [5,6], are very general but non-constructive. In a computational setting, configurations based on hyperplanes, such as those of Coatmèlec [7] and Chung and Yao [8], are preferred.

Surprisingly, the configuration of nodes introduced by Coatmèlec [7] in the plane has received several names: DH-set [2], straight line type node configuration [5], PPSN with node configuration A [9], straight line type node configuration A [10], PPSN by the recursive construction theorem using lines [11], and PPSN by line-superposition process [12].

In this paper, a new proof of the unisolvability of the interpolation problem for Coatmèlec configuration of nodes in arbitrary dimensions is presented. The proof is based on a Bézout-Radon process [13,14]. Chui and Lai [9] present a proof for the bivariate case only, state the result in arbitrary dimension, but did not prove it because of complications in their notation. Multidimensional interpolation is the basis to develop different numerical methods. The results of this paper permit to design, for example, generalized finite difference methods in irregular meshes based on Coatmèlec configuration of nodes in two [15] or more dimensions.

The contents of this paper are as follows. The definitions and notation required to set our main theorem are presented in the next section. The proof of this theorem is detailed in Section 3. Finally, in the last section, the main conclusions are summarized.

\section{Presentation of the problem}

Let $\Pi_{m}\left(\mathbb{R}^{k}\right)$ be the vector space of multivariate polynomials of degree not greater than $m$ with $k$ variables. Let $w=\left(x_{1}, \ldots, x_{k}\right)^{\top} \in \mathbb{R}^{k}$, where ${ }^{\top}$ denotes transpose, $\mathbb{N}_{0}=\mathbb{N} \cup\{0\}, j=\left(j_{1}, \ldots, j_{k}\right)^{\top} \in \Gamma:=\mathbb{N}_{0}^{k},|j|=j_{1}+\cdots+j_{k}$, $w^{j}=x_{1}^{j_{1}} x_{2}^{j_{2}} \cdots x_{k}^{j_{k}}$, and $\Gamma_{m}:=\{j \in \Gamma:|j| \leq m\}$. The set of multivariate monomials $\left\{w^{j}\right\}_{j \in \Gamma_{m}}$ is a basis of $\Pi_{m}\left(\mathbb{R}^{k}\right)$, i.e., every polynomial $p_{m}(w)$ may be written uniquely as $\sum_{j \in \Gamma_{m}} a_{j} w^{j}$, with $a_{j} \in \mathbb{R}$. Hence, the vector space $\Pi_{m}\left(\mathbb{R}^{k}\right)$ has dimension $N=C_{k+m}^{k}$, where $C_{n}^{k}$ is the binomial coefficient $\left(\begin{array}{l}n \\ k\end{array}\right)$.

Let $\Gamma^{s}:=\left\{j \in \Gamma_{m}:|j|=s\right\}, s=0,1, \ldots, m$. Note that $\Gamma_{m}=\cup_{s=0}^{m} \Gamma^{s}$, the cardinal $\# \Gamma^{s}=C_{k-1+s}^{k-1}$, and $\# \Gamma_{m}=\sum_{s=0}^{m} C_{k-1+s}^{k-1}=N$. The set of $s$ th degree monomials may be represented as a column vector of length $\# \Gamma^{s}$ 
given by $w^{(s)}:=\left(x_{1}^{s}, x_{1}^{s-1} x_{2}^{1}, \ldots, x_{i_{1}} x_{i_{2}} \cdots x_{i_{s}}, \ldots, x_{k-1}^{1} x_{k}^{s-1}, x_{k}^{s}\right)^{\top}$, for all $i=$ $\left(i_{1}, \ldots, i_{s}\right)^{\top} \in \mathbb{N}_{0}^{s}$, and $1 \leq i_{1} \leq i_{2} \leq \cdots \leq i_{s} \leq k$. Note that $w^{(0)}=(1) \in$ $\mathbb{R}, w^{(1)}=w \in \mathbb{R}^{k}$, and each component of the vector $w^{(s)}$ corresponds to a unique monomial $w^{j}$ with $j \in \Gamma^{s}$. Using this notation, every polynomial $p_{m}(w) \in \Pi_{m}\left(\mathbb{R}^{k}\right)$ may be written as $\sum_{s=0}^{m} \sum_{j \in \Gamma^{s}} a_{j} w^{j}$.

Here on, a node refers to a point in $\mathbb{R}^{k}$ and a configuration of nodes $(\mathrm{CN})$ is a set of pairwise distinct nodes $X_{m}=\left\{w_{i}\right\}_{i=1}^{N}$ where $w_{i} \equiv\left(x_{(1, i)}, x_{(2, i)}, \ldots, x_{(k, i)}\right)^{\top}$ $\in \mathbb{R}^{k}$.

The Lagrange interpolation problem may be stated as follows: Given a CN $X_{m}$ and an arbitrary set of real numbers $\left\{f_{i} \in \mathbb{R}\right\}_{i=1}^{N}$, find a polynomial $p_{m}(w) \in \Pi_{m}\left(\mathbb{R}^{k}\right)$ such that

$$
p_{m}\left(w_{i}\right):=\sum_{j \in \Gamma_{m}} a_{j} w_{i}^{j}=f_{i}, \quad i=1,2, \ldots, N .
$$

This problem is properly posed with respect to $X_{m}$ if it has a unique solution (unisolvability) for every set $\left\{f_{i}\right\}_{i=1}^{N}$. Compared with the one-dimensional case where the solvability is always assured, the solvability of multivariate interpolation depends strongly on the geometrical distribution of the nodes. A $\mathrm{CN} X_{m}$ is said to be a properly posed set of nodes (PPSN) if the Lagrange interpolation problem is properly posed with respect to $X_{m}$.

Equation (1) is a system of $N$ linear equations with a multivariate Vandermonde matrix $V_{m}$, i.e., $\left(V_{m}\right)_{i j}=w_{i}^{j}$, where $j \in \Gamma_{m}, w_{i} \in X_{m}$, and $1 \leq i \leq N$. Note that this matrix looks a little bit bizarre since rows and columns are indexed by different structural entities. A graded lexicographical order in the set of multiindices $\Gamma_{m}$ may be introduced to enhance the notation (see Ref. [16]) but this is not required in this paper.

The following theorem summarizes some previously known results.

Theorem 1 Let $X_{m}=\left\{w_{i}\right\}_{i=1}^{N}$ be a $C N$ in $k$ dimensions and $V_{m}$ the corresponding multivariate Vandermonde matrix, then the following expressions are equivalent:

(i) $X_{m}$ is a PPSN in $\mathbb{R}^{k}$.

(ii) $V_{m}$ is a nonsingular matrix, i.e., $\operatorname{det}\left(V_{m}\right) \neq 0$.

(iii) $\operatorname{rank}\left(V_{m}\right)=N$.

Let $X_{m} \equiv X_{(m, k)}=\left\{w_{i}\right\}_{i=1}^{N} \subset \mathbb{R}^{k}$ be a CN with $N=C_{m+k}^{k}$ nodes in $k$ dimensions. Let us define by induction on $k$ the following $\mathrm{CNs}$, first introduced by Coatmèlec [7,9].

Definition $2 A C N X_{m} \equiv X_{(m, k)} \subset \mathbb{R}^{k}$ is Coatmèlec in $k$ dimensions if $X_{(m, k)}=\bigcup_{p=0}^{m} X_{(p, k-1)}$ with $\# X_{(p, k-1)}=C_{p+k-1}^{k-1}$ and there exists $m+1$ hyper- 
planes $\gamma_{0}, \gamma_{1}, \ldots, \gamma_{m}$ such that $X_{(m, k-1)} \subset \gamma_{m}$ and $X_{(p, k-1)} \subset \gamma_{p} \backslash \bigcup_{q=p+1}^{m} \gamma_{q}$, for $0 \leq p \leq m-1$, with each $X_{(p, k-1)}$ being Coatmèlec in $(k-1)$ dimensions by identifying each hyperplane $\gamma_{p}$ with $\mathbb{R}^{k-1}$.

Note that, in one dimension, every $\mathrm{CN} X_{m} \equiv X_{(m, 1)} \subset \mathbb{R}$ is Coatmèlec because all its nodes are pairwise distinct, i.e., $w_{i} \neq w_{j}$, if $i \neq j$. Note also that, in Definition 2, only one node belongs to the hyperplane $\gamma_{m}$.

The main result of this paper is a proof of the following theorem.

Theorem 3 Every Coatmèlec $C N X_{m}$ in $k$ dimensions is a properly posed set of nodes in $\mathbb{R}^{k}$.

\section{Proof of the main theorem}

Our proof makes use of the following lemmas.

Lemma 4 Let us take the $C N X_{m}$ where the nodes $\left\{w_{i}\right\}_{i=1}^{N}$ are represented as column vectors in $\mathbb{R}^{k}$, and the $C N \hat{X}_{m}$ whose nodes are $\hat{w}_{i}=w_{0}+H w_{i}$, $i=1, \ldots, N$, where $w_{0}$ is an arbitrary vector and $H$ is a non-singular matrix of dimension $k$. Let $V_{m}$ and $\hat{V}_{m}$ be the Vandermonde matrices associated to the CNs $X_{m}$ and $\hat{X}_{m}$, respectively. If $\operatorname{rank}\left(V_{m}\right)=N$, then $\operatorname{rank}\left(\hat{V}_{m}\right)=N$.

Proof of Lemma 4. For every set of real numbers $\left\{f_{i} \in \mathbb{R}\right\}_{i=1}^{N}$, there exists one and only one interpolating polynomial such that $\hat{p}_{m}\left(\hat{w}_{i}\right)=f_{i}$, given by $\hat{p}_{m}(\hat{x})=p_{m}\left(H^{-1}\left(x-w_{0}\right)\right)$ where $p_{m}(x)$ is the unique interpolating polynomial for $X_{m}$ given by Theorem 1 . Therefore, $\operatorname{rank}\left(\hat{V}_{m}\right)=N$.

Lemma 5 Let $\left\{\hat{x}_{i}: i=1, \ldots, k\right\}$ be an orthonormal basis of $\mathbb{R}^{k}$, and $n_{1}$ an arbitrary vector. There always exists an orthogonal matrix $H$, representing a rotation in $\mathbb{R}^{k}$, which transform the vector $\hat{x}_{1}$ onto $H \hat{x}_{1}=\hat{n}_{1}=n_{1} /\left\|n_{1}\right\|$.

Proof of Lemma 5. If $\hat{n}_{1}=\hat{x}_{1}$, then $H=I$, the identity matrix. Otherwise, let us apply the procedure of Gram-Schmidt orthonormalization to vectors $\left\{\hat{x}_{1}, n_{1}\right\}$, yielding

$$
\hat{q}_{1}=\hat{x}_{1}, \quad q_{2}=n_{1}-\left(n_{1} \cdot \hat{q}_{1}\right) \hat{q}_{1}, \quad \hat{q}_{2}=\frac{q_{2}}{\sqrt{q_{2} \cdot q_{2}}}=\frac{q_{2}}{\left\|q_{2}\right\|},
$$

where the dot is the ordinary Euclidean dot product. An arbitrary vector $q$ can be written as $q=q_{\perp}+q_{\|}$, where $q_{\|}=\left(q \cdot \hat{q}_{1}\right) \hat{q}_{1}+\left(q \cdot \hat{q}_{1}\right) \hat{q}_{1}=Q Q^{\top} q$, where $Q=\left[\hat{q}_{1} ; \hat{q}_{2}\right]$ is the rectangular matrix whose columns are the vectors $\hat{q}_{i}$; note that $Q^{\top} Q$ is the identity matrix of dimension 2 . Taking the vector $q_{\perp}=q-q_{\|}$ as the rotation axis for the rotation matrix $H$ results in $H q=q_{\perp}+H q_{\|}=$ 
$\left(I-Q Q^{\top}\right) q+Q R Q^{\top} q$, where $R$ is the standard two-dimensional rotation matrix

$$
R=\left(\begin{array}{cc}
\cos \theta & -\sin \theta \\
\sin \theta & \cos \theta
\end{array}\right), \quad \cos \theta=\hat{x}_{1} \cdot \hat{n}_{1}, \quad \sin \theta=\sqrt{1-\left(\hat{x}_{1} \cdot \hat{n}_{1}\right)^{2}}
$$

Hence, $H=I-Q Q^{\top}+Q R Q^{\top}$ is a rotation matrix $\left(H H^{\top}=H^{\top} H=I\right.$ and $\operatorname{det}(H)=1)$ such that $H \hat{x}_{1}=\hat{n}_{1}$.

Proof of Theorem 3. Let us use the induction principle over $m$ and $k$. Let us first consider $m=0$ and any $k \in \mathbb{N}$. Clearly $X_{0}=w_{1}$ and $\operatorname{rank}\left(V_{0}\right)=1=N$. We consider next $k=1$ and $m \neq 0$. The corresponding $\mathrm{CN}$ is Coatmèlec in one dimension and the coefficient matrix is a (one-dimensional) Vandermonde matrix with maximal rank $C_{m+1}^{1}=m+1=N$, since the nodes are pairwise distinct.

By the induction hypothesis, let us assume that the theorem holds for either $m-1$ or $k-1$, and let us prove that it holds for $m$ and $k$. Here on, let us take $n=m+k$. Since $X_{m}$ is a Coatmèlec $\mathrm{CN}$ in $k$ dimensions, the following conditions are fulfilled

$$
\begin{aligned}
X_{(m, k-1)}= & \left\{w_{1}, w_{2}, \ldots, w_{C_{n-1}^{k-1}}\right\} \subset \gamma_{m}, \\
X_{(m-1, k-1)}= & \left\{w_{C_{n-1}^{k-1}+1}, \ldots, w_{C_{n-1}^{k-1}+C_{n-2}^{k-1}}\right\} \subset \gamma_{m-1} \backslash \gamma_{m}, \\
X_{(m-2, k-1)}= & \left\{w_{C_{n-1}^{k-1}+C_{n-2}^{k-1}+1}, \ldots, w_{C_{n-1}^{k-1}+C_{n-2}^{k-1}+C_{n-3}^{k-1}}\right\} \\
& \subset \gamma_{m-2} \backslash \gamma_{m-1} \cup \gamma_{m}, \\
\vdots & \\
X_{(0, k-1)}= & \left\{w_{N}\right\} \subset \gamma_{0} \backslash \gamma_{1} \cup \cdots \cup \gamma_{m},
\end{aligned}
$$

where

$$
X_{m}=X_{(m, k-1)} \cup X_{(m-1, k-1)} \cup \cdots \cup X_{(0, k-1)} .
$$


The multivariate Vandermonde matrix associated to the Lagrange interpolation problem in the $\mathrm{CN} X_{m}$ may be written as

$$
V_{m}=\left(\begin{array}{cccc}
1 & 1 & \cdots & 1 \\
& & & \\
w_{1}^{(1)} & w_{2}^{(1)} & \cdots & w_{C_{n}^{k}}^{(1)} \\
& & & \\
w_{1}^{(2)} & w_{2}^{(2)} & \cdots & w_{C_{n}^{k}}^{(2)} \\
& & & \\
\vdots & \vdots & & \vdots \\
w_{1}^{(m)} & w_{2}^{(m)} & \cdots & w_{C_{n}^{k}}^{(m)}
\end{array}\right) .
$$

Let us apply the affine transformation $\hat{w}=w_{0}+H w$ to all the nodes of the $\mathrm{CN}$, where $H$ is the orthogonal matrix given in Lemma 5, that transforms the $x_{k}$ coordinate axis in $\mathbb{R}^{k}$ into the normal vector to the hyperplane $\gamma_{m}$, and $w_{0}$ is the distance between the intersection point of the (new) rotated $x_{k}$ axis and the hyperplane $\gamma_{m}$.

The application of the affine transformation nullifies the $k$-th coordinates of the vectors $\left\{\hat{w}_{1}, \hat{w}_{2}, \ldots, \hat{w}_{C_{n-1}^{k-1}}\right\}$, hence $\hat{w}_{i}=\left(\hat{x}_{(1, i)}, \hat{x}_{(2, i)}, \cdots, \hat{x}_{(k-1, i)}, 0\right)^{\top}$. Let $\hat{V}_{m}$, where $\left(\hat{V}_{m}\right)_{i j}=\hat{w}_{i}^{j}$, be the coefficient matrix of the transformed linear system of equations. From Lemma $4, \operatorname{rank}\left(V_{m}\right)=\operatorname{rank}\left(\hat{V}_{m}\right)$.

The rows and columns of the matrix $\hat{V}_{m}$ may be sorted by renaming the nodes $\hat{w}_{i}$ to $\tilde{w}_{i}$, in order to group all its zero elements into its left-bottom part. This process preserves the rank. The resulting matrix $\tilde{V}_{m}$ has the following structure

$$
\left(\begin{array}{cc}
A & B \\
0 & A^{\prime} D
\end{array}\right)
$$


where $A$ is the $C_{n-1}^{k-1} \times C_{n-1}^{k-1}$ matrix given by

$$
A=\left(\begin{array}{cccc}
1 & 1 & \cdots & 1 \\
& & & \\
\tilde{w}_{1}^{(1)} & \tilde{w}_{2}^{(1)} & \cdots & \tilde{w}_{C_{n-1}^{k-1}}^{(1)} \\
& & & \\
\tilde{w}_{1}^{(2)} & \tilde{w}_{2}^{(2)} & \cdots & \tilde{w}_{C_{n-1}^{k-1}}^{(2)} \\
& & & \\
\vdots & \vdots & \ddots & \vdots \\
\tilde{w}_{1}^{(m)} & \tilde{w}_{2}^{(m)} & \cdots & \tilde{w}_{C_{n-1}^{k-1}}^{(m)}
\end{array}\right),
$$

$B$ is the $C_{n-1}^{k-1} \times C_{n-1}^{k}$ matrix

$$
B=\left(\begin{array}{cccc}
1 & 1 & \cdots & 1 \\
\tilde{w}_{C_{n-1}^{k-1}+1}^{(1)} & \tilde{w}_{C_{n-1}^{k-1}+2}^{(1)} & \cdots & \tilde{w}_{C_{n}^{k}}^{(1)} \\
\tilde{w}_{C_{n-1}^{k-1}+1}^{(2)} & \tilde{w}_{C_{n-1}^{k-1}+2}^{(2)} & \cdots & \tilde{w}_{C_{n}^{k}}^{(2)} \\
& & & \\
\vdots & \vdots & \ddots & \vdots \\
\tilde{w}_{C_{n-1}^{(m)}+1}^{(m)} & \tilde{w}_{C_{n-1}^{k-1}+2}^{(m)} & \cdots & \tilde{w}_{C_{n}^{k}}^{(m)}
\end{array}\right),
$$


$D$ is the $C_{n-1}^{k} \times C_{n-1}^{k}$ diagonal matrix

$$
D=\left(\begin{array}{cccc}
\hat{x}_{\left(k, C_{n-1}^{k-1}+1\right)} & 0 & \cdots & 0 \\
& & & \\
0 & \hat{x}_{\left(k, C_{n-1}^{k-1}+2\right)} & \cdots & 0 \\
\vdots & \vdots & \ddots & \vdots \\
0 & 0 & \cdots & \hat{x}_{\left(k, C_{n}^{k}\right)}
\end{array}\right),
$$

$A^{\prime}$ is the $C_{n-1}^{k} \times C_{n-1}^{k}$ matrix given by

$$
A^{\prime}=\left(\begin{array}{cccc}
1 & 1 & \cdots & 1 \\
\tilde{w}_{C_{n-1}^{k-1}+1}^{(1)} & \tilde{w}_{C_{n-1}^{k-1}+2}^{(1)} & \cdots & \tilde{w}_{C_{n}^{k}}^{(1)} \\
\vdots & \vdots & \ddots & \vdots \\
& & & \\
\tilde{w}_{C_{n-1}^{k-1}+1}^{(m)} & \tilde{w}_{C_{n-1}^{k-1}+2}^{(m)} & \cdots & \tilde{w}_{C_{n}^{k}}^{(m)}
\end{array}\right),
$$

and finally 0, cf. Eq. (2), represents the null matrix of dimensions $C_{n-1}^{k} \times C_{n-1}^{k-1}$. We recall that $C_{n}^{k}=C_{n-1}^{k-1}+C_{n-1}^{k}$.

The square matrix $A$ is a multivariate Vandermonde matrix in $(k-1)$ variables and the $C_{n-1}^{k-1}$ nodes $\left\{\tilde{w}_{i}\right\}$ are a Coatmèlec $\mathrm{CN}$ in $(k-1)$ dimensions. Therefore, by the induction hypothesis, $\operatorname{rank}(A)=C_{n-1}^{k-1}$.

The diagonal matrix $D$ is nonsingular, i.e., $\hat{x}_{k, i} \neq 0$, for $i=C_{n-1}^{k-1}+1, \ldots, C_{n}^{k}$, because if there existed at least an $i$ with $\hat{x}_{k, i}=0$, then there would be at least $C_{n-1}^{k-1}+1$ different nodes lying in the hyperplane $\gamma_{m}$, but this is not possible because $X_{m}$ is a Coatmèlec $\mathrm{CN}$. Hence, $\operatorname{rank}\left(A^{\prime} D\right)=\operatorname{rank}\left(A^{\prime}\right)$. Moreover, the matrix $A^{\prime}$ is also a multivariate Vandermonde matrix corresponding to the $C_{n-1}^{k}$ nodes that do not belong to the hyperplane $\gamma_{m}$. Since the Coatmèlec property of a $\mathrm{CN}$ does not change under either rotation or translation of all the nodes, the $\mathrm{CN}\left\{\tilde{w}_{i}\right\}, i=C_{n-1}^{k-1}+1, \cdots, C_{n}^{k}$, is also a Coatmèlec $\mathrm{CN}$. The induction hypothesis yields that the rank of matrix $A^{\prime}$ is $C_{n-1}^{k}$.

Finally, the rank of the $C_{n}^{k} \times C_{n}^{k}$ matrix $\tilde{V}_{m}$ is $\operatorname{rank}(A)+\operatorname{rank}\left(A^{\prime}\right)=C_{n-1}^{k-1}+$ $C_{n-1}^{k}=C_{n}^{k}$, and the theorem is proved. 


\section{Conclusions}

The unisolvency of the problem of multivariate polynomial interpolation in a Coatmèlec CN, a kind of properly posed set of nodes defined by hyperplanes, has been shown through a new and straightforward proof. This proof uses elementary techniques from linear algebra. This fact permits the understanding of the topic by nonexperts and opens the possibility of it being incorporated in numerical analysis textbooks.

The geometrical condition characterizing Coatmèlec CNs is one of the most general conditions currently available for the characterization of properly posed set of nodes defined by hperplanes, which is easier and more efficient to be checked by an automatic computational software than the widely known geometrical characterization of Chung and Yao [8]. Therefore, Coatmèlec CNs are useful in mesh generation for the numerical solution of partial differential equations in irregular domains, such as generalized finite difference methods.

\section{Acknowledgements}

The authors thank to Drs. Mariano Gasca and Juan I. Ramos for pointing us some references and for their useful comments which have greatly improved the presentation. The authors also thank a reviewer for pointing out a mis-

take in the original proof of Lemma 5. The research reported in this paper was partially supported by Project MTM2010-19969 from the Ministerio de Ciencia e Innovación of Spain and Grant PAID-06-09-2734 from the Universidad Politécnica de Valencia.

\section{References}

[1] M. GASCA AND T. SAUER, On the history of multivariate polynomial interpolation, J. Comput. Appl. Math., 122 (2000), pp. 23-35.

[2] M. Gasca And T. Sauer, Polynomial interpolation in several variables, Advances Comput. Math., 12 (2000), pp. 377-410.

[3] R.A. Lorentz, Multivariate Hermite interpolation by algebraic polynomials: A survey, J. Comput. Appl. Math., 122 (2000), pp. 167-201.

[4] L. Bos, On certain configurations of points in $\mathbb{R}^{n}$ which are unisolvent for polynomial interpolation, J. Approx. Theor., 64 (1991), pp. 271-280. 
[5] X.-Z. Liang, C.-M. LÜ, And R.-Z. Feng, Properly posed sets of nodes for multivariate Lagrange interpolation in $C^{s}$, SIAM J. Numer. Anal., 39 (2001), pp. 587-595.

[6] X.-Z. Liang, R.-H. Wang, L.-H. Cui, J.-L. Zhang, and M. Zhang, Some researches on trivariate Lagrange interpolation, J. Comput. Appl. Math., 195 (2006) pp. 192-205.

[7] C. Contmèlec, Approximation et interpolation des fonctions différentiables de plusieurs variables, Ann. Sci. École Norm. Sup., 83 (1966), pp. 271-341.

[8] K. C. Chung And T. H. YaO, On lattices admitting unique Lagrange interpolation, SIAM J. Numer. Anal., 14 (1977), pp. 735-743.

[9] C. K. Chui And M. J. LAI, Vandermonde determinant and Lagrange interpolation in $R^{s}$, in Nonlinear and Convex Analysis, B. L. Lin and S. Simon, eds., Marcel Dekker, New York, 1987, pp. 23-35.

[10] P. ZHU, On Birkhoff interpolation by polynomials in several variables, J. Comput. Appl. Math., 85 (1997), pp. 263-270.

[11] X.-Z. LiAng AND C.-M. Lu, Properly posed set of nodes for bivariate Lagrange interpolation, in Approximation Theory IX, Volume 1: Theoretical Aspects, C.K. Chui and L.L. Schumacker, eds., Vanderbilt University Press, Nashville, TN, 1998, pp. 189-196.

[12] X.-Z. Liang, L.-H. Cui, And J.-L. Zhang, The application of CayleyBacharach theorem to bivariate Lagrange interpolation, J. Comput. Appl. Math., 163 (2004), pp. 177-187.

[13] J. RAdon, Zur mechanischen Kubatur, Monatshefte für Mathematik, 52 (1948), pp. 286-300.

[14] R. B. Guenther and E. L. Roetman, Some observations on interpolation in higher dimensions, Math. Comput., 24 (1970), pp. 517-522.

[15] García-March, M.A., Arevalillo-Herráez, M., Villatoro, F.R., Giménez, F., And Fernández De Córdoba, P., A generalized finite difference method using Coatmèlec lattices, Comput. Phys. Comm., 180 (2009) pp. 1125-1133.

[16] D. A. Cox, J. B. Little, And D. B. O'SheA, Ideals, Varieties, and Algorithms: An Introduction to Computational Algebraic Geometry and Commutative Algebra, Second ed., Springer Verlag, New York, 1996. 